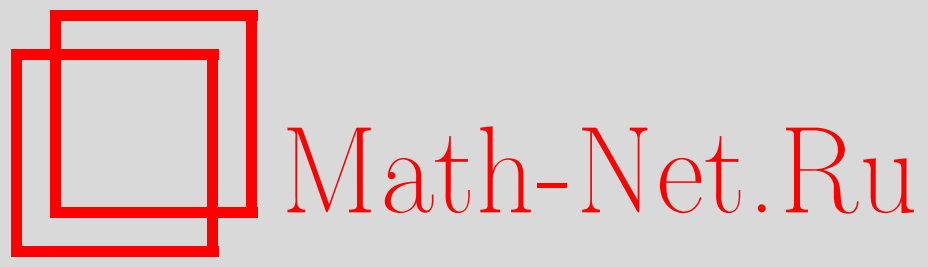

В. Г. Михайлов, Предельные теоремы пуассоновского типа для числа неполных совпадений $s$-цепочек, Теория вероятн. и ее примен., 2002, том 47, выпуск 2, 350-357

DOI: https://doi.org/10.4213/tvp3654

Использование Общероссийского математического портала MathNet.Ru подразумевает, что вы прочитали и согласны с пользовательским соглашением

http://www.mathnet.ru/rus/agreement

Параметры загрузки:

IP : 54.157 .27 .8

26 апреля 2023 г., $11: 39: 13$

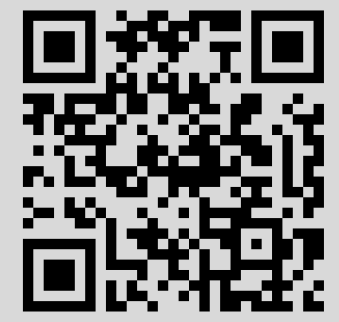




\title{
ПРЕДЕЛЬНЫЕ ТЕОРЕМЫ ПУАССОНОВСКОГО ТИПА ДЛЯ ЧИСЛА НЕПОЛНЫХ СОВПАДЕНИЙ $S$-ЦЕПОЧЕК ${ }^{1)}$
}

\begin{abstract}
Основной результат статьи - предельная теорема для вектора из чисел неполных (с заданным числом несовпавших букв) совпадений $s$-цепочек в двух последовательностях независимых одинаково распределенных случайных величин. Указаны вид предельного совместного распределения и достаточные условия сходимости. Получены явные оценки близости к сопровождаюшему многомерному сложному пуассоновскому распределению. При доказательстве использован локальный вариант метода Чена-Стейна оценивания точности пуассоновской аппроксимации для распределения набора зависимых случайных индикаторов.
\end{abstract}

Ключевые слова и фразы: совпадения и повторения цепочек, неполные совпадения, предельное сложное пуассоновское распределение, явные оценки скорости сходимости, метод Чена-Стейна.

1. Введение. Пусть $X=\left(X_{1}, \ldots, X_{m}\right)$ и $Y=\left(Y_{1}, \ldots, Y_{n}\right)$ - две последовательности независимых одинаково (внутри каждой последовательности) распределенных случайных величин, принимающих значения в множестве $\{1,2, \ldots\}$. Введем обозначения

$$
\begin{aligned}
p_{k} & =\mathbf{P}\left\{X_{1}=k\right\}, \quad q_{k}=\mathbf{P}\left\{Y_{1}=k\right\}, \quad k=1,2, \ldots, \\
p & =\max _{k} p_{k} I\left(q_{k}>0\right), \quad q=\max _{k} q_{k} I\left(p_{k}>0\right)
\end{aligned}
$$

(здесь и далее выражение $I(\cdot)$ обозначает индикатор случайного события или неслучайного множества), $r=\max _{k} p_{k} q_{k}, R=\sum_{k=1}^{\infty} p_{k} q_{k}$.

На множестве цепочек $x=\left(x_{1}, \ldots, x_{s}\right), x_{i} \in \mathbf{Z}^{+}, s=1,2, \ldots$, введем функцию $H(x, y)=\sum_{i=1}^{s} I\left(x_{i}=y_{i}\right)$. Рассмотрим события

$$
\begin{aligned}
& H_{i, j}(s, c)=\left\{H\left(\left(X_{i}, \ldots, X_{i+s-1}\right),\left(Y_{j}, \ldots, Y_{j+s-1}\right)\right)=c\right\}, \\
& H_{i, j}^{\prime}(s, c)=H_{i, j}(s, c) \cap\left\{X_{i}=Y_{j}\right\} \cap\left\{X_{i+s-1}=Y_{j+s-1}\right\},
\end{aligned}
$$

которые будем называть $(s, c)$-совпадениями и $(s, c)^{\prime}$-совпадениями с координатами $i$ и $j$. При $c=s$ оба этих понятия имеют один и тот же смысл полного совпадения цепочек. При $c<s$ мы используем термин неполные совпадения.

Число $(s, c)$-совпадений и число $(s, c)^{\prime}$-совпадений цепочек последовательностей $X$ и $Y$ выражаются формулами

$$
N(s, c)=\sum_{i=1}^{m} \sum_{j=1}^{n} I\left(H_{i, j}(s, c)\right), \quad N^{\prime}(s, c)=\sum_{i=1}^{m} \sum_{j=1}^{n} I\left(H_{i, j}^{\prime}(s, c)\right) .
$$

В этом определении считается, что $X_{m+i}=X_{i}, Y_{n+i}=Y_{i}, i=1,2, \ldots$.

Ниже с помошью локального варианта метода Чена-Стейна для совместного распределения зависимых случайных индикаторов будет доказана

* Математический институт им. В. А. Стеклова РАН, ул. Губкина, 8, 117966 Москва, ГСП-1, Россия.

1) Работа выполнена при поддержке Российского фонда фундаментальных исследований (проект 99-01-00012) и Совета по грантам Президента РФ и государственной подцержке ведущих научных школ (проект 00-15-96136). 
Теорема 1. Пусть параметры $m, n \rightarrow \infty$, величины $s$ и с постоянны, причем $2 \leqslant c \leqslant s$, а вероятности $p_{k}, q_{k}$ меняются так, что

$$
\lambda(m, n)=m n R^{c} \rightarrow \lambda<\infty, \quad m p^{c}+n q^{c}+r R^{-1} \rightarrow 0 .
$$

Тогда случайные величины $N^{\prime}(k, c), k=c, \ldots, s$, асимптотически независимы, а их распределения сходятся $к$ распределениям Пуассона с параметрами $\lambda_{k}=C_{k-2}^{c-2} \lambda$ соответственно.

3 а м е ч а н и е 1 . При условии (1)

$$
\mathbf{E} N(s, c+1) \rightarrow 0 \quad(s \geqslant c+1), \quad \mathbf{E} N(s, c-1) \rightarrow \infty \quad(s \geqslant c-1) .
$$

Аналогичные соотношения выполнены для $\mathbf{E} N^{\prime}(s, c \pm 1)$. Если при этом $N(s+1, c+1)=0$, то каждому $(s-k, c)^{\prime}$-совпадению отвечает своя серия из $k+1$ штук $(s, c)$-совпадений, причем каждая серия $(s, c)$-совпадений порождена каким-либо $(s-k, c)^{\prime}$-совпадением.

Эти свойства позволяют вывести из теоремы 1 следующее утверждение.

Теорема 2. Пусть выполнены условия теоремы 1. Тогда распределение ( $s-$ $c+1)$-мерного вектора $(N(l, c), l=c, \ldots, s)$ сходится $\kappa$ распределению $\operatorname{CP}\left(\lambda_{c}, \ldots, \lambda_{s}\right)$ вектора $\left(\sum_{k=c}^{l}(l-k+1) \pi_{k}, l=c, \ldots, s\right)$, где $\pi_{c}, \ldots, \pi_{s}-$ независимые случайные величинь, распределенные по закону Пуассона с параметрами $\lambda_{c}, \ldots, \lambda_{s}$ соответственно.

3 а м е ч а н и е 2. Теоремы 1 и 2 и замечание 1 дают следующую картину асимптотического поведения числа $(s, c)$-совпадений. Эти совпадения возникают сериями, число серий с вероятностью, стремящейся к единице, совпадает с числом $(s, c)^{\prime}$-совпадений и имеет в пределе распределение Пуассона с параметром $\lambda(s)=$ $\lambda \sum_{k=c}^{s} C_{k-2}^{c-2}=\lambda C_{s-1}^{c-1}$, а длины серий асимптотически независимы и имеют в пределе распределение

$$
\mathbf{P}\{\kappa=k+1\}=C_{s-k-2}^{c-2}\left(C_{s-1}^{c-1}\right)^{-1}, \quad k=0,1, \ldots .
$$

Поэтому предельным распределением для $N(s, c)$ является сложное пуассоновское распределение с производящей функцией $\exp \left\{\sum_{k=c}^{s} \lambda_{k}\left(z^{s-k+1}-1\right)\right\}$. При $c=s$ это распределение оказывается просто пуассоновским с параметром $\lambda_{s}=\lambda$.

3 а м е ч а н и е 3 . В работе [1] был использован иной прием перечисления $(s, c)$-совпадений. Каждая серия таких совпадений начинается с первого в серии $(s, c)$ совпадения - события $H_{i, j}(s, c) \cap H_{i-1, j-1}(s, c-1)$. Число «первых» совпадений равно числу серий. В работе [1] изучалось число повторений $s$-цепочек (т.е. число $(s, s)$ совпадений цепочек внутри одной последовательности). Используя свойства «первых» повторений $s$-цепочек, было показано, что число серий повторений $s$-цепочек имеет в пределе распределение Пуассона, а длины серий асимптотически независимы и имеют в пределе геометрическое распределение с параметром $\rho=\lim R$. В работе [1] допускался и случай $\rho>0$.

Продолжим изложение результатов. При доказательстве теорем 1 и 2 удалось получить оценки близости исследуемых совместных распределений к сопровождающим распределениям пуассоновского типа.

Обозначим через $\rho(F, G)$ расстояние по вариации между распределениями $F$ и $G$. Для распределений, сосредоточенных на не более чем счетном множестве $K$, оно выражается формулой

$$
\rho(F, G)=\frac{1}{2} \sum_{k \in K}|F\{k\}-G\{k\}| .
$$

Обозначим через $\operatorname{Po}\left(\lambda_{c}, \ldots, \lambda_{s}\right)$ распределение $(s-c+1)$-мерного случайного вектора, состоящего из независимых случайных величин, распределенных по закону Пуассона с параметрами $\lambda_{c}, \ldots, \lambda_{s}$ соответственно. Обозначим через $\mathscr{L}(s, c)$ и $\mathscr{L}^{\prime}(s, c)$ распределения векторов $(N(l, c), l=c, \ldots, s)$ и $\left(N^{\prime}(l, c), l=c, \ldots, s\right)$ соответственно.

Используем обозначения

$$
\lambda_{k}(m, n)=C_{k-2}^{c-2}(1-R)^{k-c} \lambda(m, n) .
$$

Заметим сразу, что $\mathbf{E} N(k, c)=\lambda_{k}(m, n)$. 
Теорема 3. Пусть параметры $m, n \geqslant 2 s-1 u 2 \leqslant c \leqslant s$. Тогда

$$
\begin{aligned}
& \rho\left(\mathscr{L}^{\prime}(s, c), \operatorname{Po}\left(\lambda_{c}(m, n), \ldots, \lambda_{s}(m, n)\right)\right) \leqslant S, \\
& \rho\left(\mathscr{L}(s, c), \operatorname{CP}\left(\lambda_{c}(m, n), \ldots, \lambda_{s}(m, n)\right)\right) \leqslant S+T,
\end{aligned}
$$

гдe

$$
\begin{aligned}
S= & (2 s-1)\left(C_{s-1}^{c-1}\right)^{2} \lambda(m, n)\left(\lambda(m, n)\left(m^{-1}+n^{-1}\right)\right. \\
& \left.+R+m p^{c}+n q^{c}+(2 s-1)\left(r R^{-1}\right)^{c}\right), \quad T=C_{s}^{c} R \lambda(m, n) .
\end{aligned}
$$

3 а м е ч а н и е 4. Аналогичные утверждения могут быть доказаны и для числа неполных повторений цепочек в одной последовательности независимых испытаний. Boпрос о виде совместных предельных распределений для $(s, c)$-совпадений цепочек в паре последовательностей и для $(s, c)$-повторений в одной последовательности был впервые поднят в 1974 году и оставался открытым до настоящего времени.

3 а м е ч а н и е 5 . Теоремы 1 и 2 являются следствиями теоремы 3. Они описывают свойства числа $(s, c)^{\prime}$-совпадений и числа $(s, c)$-совпадений при постоянных значениях параметров $s$ и $c$ и $R \rightarrow 0$ (последнее вытекает из условия (1)).

3 а м е ч а н и е 6 . Величина $N(s, s)$ - это число полных совпадений $s$-цепочек. Число полных совпадений и число полных повторений цепочек изучались, например, в [1], [2] и [3] (см. также [4]). Как мы уже упоминали в замечании 2, был охвачен и случай, когда $R \rightarrow \rho>0$. В этом случае предельное распределение величины $N(s, s)$ является сложным пуассоновским распределением с производящей функцией $\exp \{\lambda(z-1) /(1-\rho z)\}$. Этот факт свидетельствует о том, что предельное распределение для вектора $(N(s-l, c), l=0, \ldots, s-c)$ при $R \rightarrow \rho>0$ будет существенно отличаться от приведенного в теореме 2 . Автору пока не известен вид предельного распределения $N(s, c)$ при $c<s$ в этом случае. Следует заметить, что условие $R \rightarrow 0$ является сушественным в доказательстве наших теорем, и в случае $R \rightarrow \rho>0$ использованный ход доказательств, скорее всего, будет непригодным.

3 а м е ч а н и е 7 . Свойства величин $N(s, c)$ при $c \leqslant s-1$ изучались в работе [5], где была получена оценка для расстояния по вариации между распределением величины $N(s, c)$ и пуассоновским распределением. Впрочем, как показывает теорема 2, в нашем случае эти оценки особого значения не имеют. Нетрудно проверить, что (как и следовало ожидать) указанные в [5] границы для расстояния по вариации до пуассоновского распределения в условиях теоремы 2 не сходятся к нулю (при $c \geqslant 1$ ). Близкие вопросы рассматривались в статьях [6], [7], где были доказаны предельные теоремы для распределения величины $R(d)$ - длины самого длинного совпадения отрезков с заданным числом $d$ несовпадений букв.

3 а м е ч а н и е 8 . Теорема 3 позволяет сделать определенные выводы о предельном поведении величины $N(s, c)$ и при переменных $s$ и $c$. Она, например, показывает, что если $m, n, s \rightarrow \infty, \lambda_{s}(m, n) \rightarrow \mu<\infty, 2 \leqslant c \leqslant s$ и $s m^{-1}+s n^{-1}+s^{s-c+1}(R+$ $\left.m p^{c}+n q^{c}+s\left(r R^{-1}\right)^{c}\right) \longrightarrow 0$, то распределение случайной величины $N(s, c)$ сходится к распределению Пуассона с параметром $\mu$. Обратим внимание на то, что, в отличие от случая $s=$ const, здесь $\lambda(m, n) \rightarrow 0$.

3 а м е ч а н и е 9. Утверждения теорем 1 и 2 справедливы и для неполных совпадений цепочек из обычных (не замкнутых в циклы) последовательностей случайных величин. Возникаюшие в этом случае эффекты, связанные со спецификой свойств совпадений цепочек, расположенных в началах и концах последовательностей, значительно усложняют рассуждения.

3 а м е ч а н и е 10 . В одномерном случае оценка (2) теоремы 3 может быть сушественно уточнена. Пусть $L^{\prime}(s, c)$ - распределение случайной величины $N^{\prime}(s, c)$. Тогда

$$
\rho\left(L^{\prime}(s, c), \operatorname{Po}\left(\lambda_{s}(m, n)\right)\right) \leqslant \frac{1-\exp \left\{-\lambda_{s}(m, n)\right\}}{\lambda_{s}(m, n)} S
$$

Значительное уточнение допускает в одномерном случае и оценка (3) теоремы 3. Этому результату будет посвящена специальная статья.

Завершая вводную часть, приведем два интересных следствия сформулированных теорем. 
Следствие 1. Пусть параметры $m, n \rightarrow \infty$, величины $s$ и с постоянны, причем $2 \leqslant c \leqslant s$, а вероятности $p_{k}, q_{k}$ меняются так, что

$$
\lambda(m, n)=m n R^{c} \rightarrow \infty, \quad \lambda^{2} m^{-1}+\lambda^{2} n^{-1}+\lambda\left(R+m p^{c}+n q^{c}+\left(r R^{-1}\right)^{c}\right) \longrightarrow 0 .
$$

Тогда

а) случайные величины $\left(N^{\prime}(k, c)-\mathbf{E} N^{\prime}(k, c)\right)\left(\mathbf{E} N^{\prime}(k, c)\right)^{-1 / 2}, k=c, \ldots, s$, асимптотически независимы, а их распределения сходятся $\kappa$ стандартному нормальному распределению,

b) Функиия распределения вектора $\left((\lambda(m, n))^{-1 / 2}\left(N(l, c)-\lambda_{l}(m, n)\right), l=c, \ldots, s\right)$ сходится $\kappa(s-c+1)$-мерному нормальному распределению с нулевым вектором средних и матричей ковариачий

$$
\left\|\sum_{k=c}^{\min \{i, j\}}(i-k+1)(j-k+1) C_{k-2}^{c-2}\right\| .
$$

Рассмотрим величину $\nu(c)=\min \{s: N(s, c)>0\}$, выражающую минимальную длину отрезков в последовательностях $\left(X_{1}, \ldots, X_{m}\right)$ и $\left(Y_{1}, \ldots, Y_{n}\right)$, имеющих между собой ровно $c$ совпадений букв (с учетом их мест в отрезках).

Следствие 2. Пусть выполнены условия теоремь 1. Тогда

$$
\mathbf{P}\{c \leqslant \nu(c) \leqslant k\} \rightarrow 1-\exp \left\{-\lambda C_{k-1}^{c-1}\right\}, \quad k=c, c+1, \ldots
$$

2. Доказательства для $(s, c)^{\prime}$-совпадений. В этом пункте доказываются оценка (2) теоремы 3 и теорема 1.

Нам будет удобно разбить события $H_{i, j}^{\prime}(s, c)$ на более мелкие следующим образом. Рассмотрим множество $\Delta_{s}$ векторов $\delta=\left(\delta_{1}, \ldots, \delta_{s}\right)$ из нулей и единиц, у которых $\sum_{l} \delta_{l}=c$ и $\delta_{1}=\delta_{s}=1$. При $\delta \in \Delta_{s}$ положим

$$
H_{i, j}^{\prime}(s, c, \delta)=\left\{X_{i+l-1}=Y_{j+l-1} \Longleftrightarrow \delta_{l}=1, l=1, \ldots, s\right\} .
$$

Введем обозначения $W_{s}(i, j)=I\left\{H_{i, j}^{\prime}(s, c)\right\}, W(i, j, \delta)=I\left\{H_{i, j}^{\prime}(s, c, \delta)\right\}$, где $i=1, \ldots, m, j=1, \ldots, n$ и $\delta \in \Delta_{s}$. Очевидно, что $W_{s}(i, j)=\sum_{\delta \in \Delta_{s}} W(i, j, \delta)$. Надо заметить (см. [8, формула (II.12.8)]), что

$$
\sum_{k=c}^{s}\left|\Delta_{k}\right|=\sum_{k=c}^{s} C_{k-2}^{c-2}=C_{s-1}^{c-1} .
$$

Обозначим через $W$ набор случайных величин $\{W(i, j, \delta)\}_{\Gamma}$ на множестве $\Gamma=$ $\{1, \ldots, m\} \times\{1, \ldots, n\} \times \Delta$, где $\Delta=\bigcup_{k=c}^{s} \Delta_{k}$. Пусть $\pi=\{\pi(i, j, \delta)\}_{\Gamma}$ - набор независимых случайных величин, имеющих распределения Пуассона с параметрами

$$
\mathbf{E} \pi(i, j, \delta)=\mathbf{E} W(i, j, \delta)=R^{c}(1-R)^{k-c}
$$

при $\delta \in \Delta_{k}$.

Будем использовать обозначение $\mathscr{L}(Z)$ для распределения случайной величины (или набора случайных величин) $Z$.

Теорема 4. Пусть параметры $m, n \geqslant 2 s-1$. Тогда

$$
\rho(\mathscr{L}(W), \mathscr{L}(\pi)) \leqslant S
$$

где $S$ определено в (4).

Д о к а з а т е л ь с т в о. Для элементов $(i, j, \delta) \in \Gamma$ будем использовать обозначение $a=(i, j, \delta)$. Для числа компонент вектора $\delta$ будем использовать обозначение $|\delta|$. Другими словами, $\delta$ принадлежит $\Delta_{|\delta|}$.

Рассмотрим множество

$$
\Gamma_{a}^{\text {ind }}=\left\{a^{\prime} \in \Gamma: i^{\prime}-i \geqslant|\delta|, i-i^{\prime} \geqslant\left|\delta^{\prime}\right|, \text { или } j^{\prime}-j \geqslant|\delta| \text {, или } j-j^{\prime} \geqslant\left|\delta^{\prime}\right|\right\} .
$$

Нетрудно проверить, что случайная величина $W(a)$ не зависит от совокупности случайных величин $\left\{W\left(a^{\prime}\right), a^{\prime} \in \Gamma_{a}^{\text {ind }}\right\}$. Положим

$$
\Gamma_{a}=(\Gamma \backslash\{a\}) \backslash \Gamma_{a}^{\text {ind }} .
$$


Воспользуемся теоремой 10.А из книги [9], из которой следует, что

$$
\rho(\mathscr{L}(W), \mathscr{L}(\pi)) \leqslant \sum_{a \in \Gamma} \mathbf{E} W(a)(\mathbf{E} W(a)+\mathbf{E} U(a))+\sum_{a \in \Gamma} \mathbf{E} W(a) U(a),
$$

где $U(a)=\sum_{a^{\prime} \in \Gamma_{a}} W\left(a^{\prime}\right)$.

Оценим первую сумму в правой части (9). Ее можно записать в виде

$$
\sum_{a \in \Gamma} \mathbf{E} W(a)(\mathbf{E} W(a)+\mathbf{E} U(a))=\sum_{a \in \Gamma} \sum_{a^{\prime} \in\{a\} \cup \Gamma_{a}} \mathbf{E} W(a) \mathbf{E} W\left(a^{\prime}\right) .
$$

Число слагаемых во внутренней сумме допускает оценку $\left|\{a\} \cup \Gamma_{a}\right|=\left|\Gamma \backslash \Gamma_{a}^{\text {ind }}\right| \leqslant$ $\left(C_{s-1}^{c-1}\right)^{2}(m n-(m-2 s+1)(n-2 s+1))$. Кроме того, согласно (7) и (1), $\mathbf{E} W(a) \leqslant$ $R^{c}=m^{-1} n^{-1} \lambda(m, n)$. Значит,

$$
\sum_{a \in \Gamma} \mathbf{E} W(a)(\mathbf{E} W(a)+\mathbf{E} U(a)) \leqslant\left(C_{s-1}^{c-1}\right)^{2}(2 s-1) \lambda^{2}(m, n)\left(m^{-1}+n^{-1}\right) .
$$

Оценим вторую сумму в правой части (9). Введем множества

$$
\begin{aligned}
& \Gamma_{a}^{s}=\left\{a^{\prime}=\left(i^{\prime}, j^{\prime}, k^{\prime}\right) \in \Gamma_{a}:-\left|\delta^{\prime}\right|<i^{\prime}-i=j^{\prime}-j<|\delta|\right\}, \\
& \Gamma_{a}^{*}= \Gamma_{a} \backslash \Gamma_{a}^{s}, \\
& \Gamma_{a}^{* *}=\left\{a^{\prime} \in \Gamma_{a}^{*}: 0<i^{\prime}-i<|\delta| \text { или } 0<i-i^{\prime}<\left|\delta^{\prime}\right|,\right. \\
&\left.0<j^{\prime}-j<|\delta| \text { или } 0<j-j^{\prime}<\left|\delta^{\prime}\right|\right\} .
\end{aligned}
$$

Лемма 1. Пусть $a \in \Gamma$.

(a) $\Pi$ ри $a^{\prime} \in \Gamma_{a}^{s}$ имеет место неравенство $\mathbf{E} W(a) W\left(a^{\prime}\right) \leqslant R \mathbf{E} W(a)$.

(b) При $a^{\prime} \in \Gamma_{a}^{*} \backslash \Gamma_{a}^{* *}$ выполнены неравенства

$\mathbf{E} W(a) W\left(a^{\prime}\right) \leqslant q^{c} \mathbf{E} W(a)$, если $0<i^{\prime}-i<|\delta|$ или $0<i-i^{\prime}<\left|\delta^{\prime}\right|$,

$\mathbf{E} W(a) W\left(a^{\prime}\right) \leqslant p^{c} \mathbf{E} W(a)$, если $0<j^{\prime}-j<|\delta|$ или $0<j-j^{\prime}<\left|\delta^{\prime}\right|$.

(c) При а $a^{\prime} \in \Gamma_{a}^{* *}$ имеет место неравенство $\mathbf{E} W(a) W\left(a^{\prime}\right) \leqslant r^{c}$.

Д о к а з а т е л ь с т в о. (а) Пусть $a^{\prime} \in \Gamma_{a}^{s}$ и, для определенности, $i^{\prime}<i$. Тогда

$$
\mathbf{E} W(a) W\left(a^{\prime}\right) \leqslant \mathbf{E}\left(W(a) I\left\{X_{i^{\prime}}=Y_{j^{\prime}}\right\}\right)=\mathbf{E}\left(W(a) \mathbf{P}\left\{X_{i^{\prime}}=Y_{j^{\prime}}\right\}\right)=R \mathbf{E} W(a) .
$$

(b) Пусть $a^{\prime} \in \Gamma_{a}^{*} \backslash \Gamma_{a}^{* *}$ и, для определенности, $0<i^{\prime}-i<|\delta|$. Тогда $j^{\prime}-j>|\delta|$ или $j^{\prime}-j<-\left|\delta^{\prime}\right|$. Поэтому $\mathbf{E}\left(W\left(a^{\prime}\right) \mid A\right) \leqslant q^{c}$ для любого события $A$ с $\mathbf{P}(A)>0$, порожденного случайными величинами $X_{i}, \ldots, X_{i+|\delta|-1}$ и $Y_{j}, \ldots, Y_{j+|\delta|-1}$. Поэтому

$$
\mathbf{E} W(a) W\left(a^{\prime}\right) \leqslant \mathbf{E}\left(W(a) \mathbf{E}\left(W\left(a^{\prime}\right) \mid W(a)=1\right)\right) \leqslant q^{c} \mathbf{E} W(a) .
$$

(c) Воспользуемся схемой доказательства леммы 2 из работы [1]. Выберем некоторые $a=(i, j, \delta) \in \Gamma$ и $a^{\prime}=\left(i^{\prime}, j^{\prime}, \delta^{\prime}\right) \in \Gamma_{a}^{* *}$. Рассмотрим двудольный граф $G\left(a, a^{\prime}\right)$ с множеством вершин

$$
\begin{aligned}
V & =V_{1} \cup V_{2}, \quad V_{1}=\left\{i+l-1: \delta_{l}=1\right\} \cup\left\{i^{\prime}+l-1: \delta^{\prime}=1\right\}, \\
V_{2} & =\left\{j+l-1: \delta_{l}=1\right\} \cup\left\{j^{\prime}+l-1: \delta^{\prime}=1\right\}
\end{aligned}
$$

и множеством из $2 c$ ребер $E=\left\{(i+l-1, j+l-1): \delta_{l}=1\right\} \cup\left\{\left(i^{\prime}+l-1, j^{\prime}+l-1\right): \delta_{l}^{\prime}=1\right\}$. $\Gamma$ раф $G\left(a, a^{\prime}\right)$ не имеет циклов и состоит из связных компонент, представляюших собой простые цепи, в которых чередуются вершины из $V_{1}$ и $V_{2}$. Обозначим через $N_{s_{1}, s_{2}}$ число цепей с $s_{1}$ вершинами в $V_{1}$ и с $s_{2}$ вершинами в $V_{2},\left|s_{1}-s_{2}\right| \leqslant 1$. Цепь с $s_{1}$ вершинами в $V_{1}$ и с $s_{2}$ вершинами в $V_{2}$ имеет $s_{1}+s_{2}-1$ ребер, поэтому

$$
\sum_{s_{1}, s_{2}}\left(s_{1}+s_{2}-1\right) N_{s_{1}, s_{2}}=2 c .
$$


Как нетрудно проверить, обратившись к определению случайной величины $W(a)$,

$$
\mathbf{E} W(a) W\left(a^{\prime}\right) \leqslant \prod_{s_{1}, s_{2}}\left(\sum_{k=1}^{\infty} p_{k}^{s_{1}} q_{k}^{s_{2}}\right)^{N\left(s_{1}, s_{2}\right)} .
$$

Заметим, что

$$
\begin{aligned}
\sum_{k} p_{k}^{h} q_{k}^{h} & \leqslant r^{h-1 / 2} \sum_{k}\left(p_{k} q_{k}\right)^{1 / 2} \leqslant r^{(2 h-1) / 2} \\
\sum_{k} p_{k}^{h} q_{k}^{h+1} & \leqslant r^{h}=r^{((2 h+1)-1) / 2}, \quad \sum_{k} p_{k}^{h+1} q_{k}^{h} \leqslant r^{h}=r^{((2 h+1)-1) / 2} .
\end{aligned}
$$

Подставим эти оценки в (13) и воспользуемся равенствами (12): $\mathbf{E} W(a) W\left(a^{\prime}\right) \leqslant r^{c}$. Лемма доказана.

3 а м е ч а н и е 11 . Оценки для $\mathbf{E} W(a) W\left(a^{\prime}\right)$ типа полученных в лемме 1 используются при доказательстве предельных теорем пуассоновского типа не только методом Чена-Стейна, но и методом моментов (см., например, [1]). Близкие по смыслу к оценкам леммы 1 (и, возможно, немного более точные) оценки для случая одинаково распределенных последовательностей содержатся в лемме 4.1 работы [5].

Продолжим доказательство теоремы 4. Заметим, что в силу определений и равенства (6)

$$
\begin{gathered}
\sum_{a \in \Gamma} \mathbf{E} W(a)=C_{s-1}^{c-1} \lambda(m, n), \\
\left|\Gamma_{a}^{s}\right|=(2 s-1) C_{s-1}^{c-1}, \quad\left|\Gamma \times \Gamma_{a}^{* *}\right| \leqslant(2 s-1)^{2} m n\left(C_{s-1}^{c-1}\right)^{2},
\end{gathered}
$$

а численности двух частей множества $\Gamma_{a}^{*} \backslash \Gamma_{a}^{*}$, упомянутых в п. (b) леммы 1 , оцениваются величинами $(2 s-1) n C_{s-1}^{c-1}$ и $(2 s-1) m C_{s-1}^{c-1}$ соответственно. Поэтому, используя лемму, получаем

$$
\begin{gathered}
\sum_{a \in \Gamma} \sum_{a^{\prime} \in \Gamma_{a}^{s}} \mathbf{E} W(a) W\left(a^{\prime}\right) \leqslant(2 s-1) R\left(C_{s-1}^{c-1}\right)^{2} \lambda(m, n), \\
\sum_{a \in \Gamma} \sum_{a^{\prime} \in \Gamma_{a}^{*} \backslash \Gamma_{a}^{* *}} \mathbf{E} W(a) W\left(a^{\prime}\right) \leqslant(2 s-1)\left(C_{s-1}^{c-1}\right)^{2} \lambda(m, n)\left(m p^{c}+n q^{c}\right), \\
\sum_{a \in \Gamma} \sum_{a^{\prime} \in \Gamma_{a}^{* *}} \mathbf{E} W(a) W\left(a^{\prime}\right) \leqslant(2 s-1)^{2}\left(C_{s-1}^{c-1}\right)^{2} m n r^{c} .
\end{gathered}
$$

Подставив оценки (10) и (14) в (9), придем к неравенству (8). Теорема 4 доказана.

3 а м е ч а н и е 12 . Если бы в теореме 4 нас интересовало поведение распределения не самого набора $W$, а лишь суммы всех или некоторой части его элементов, например, величины $N^{\prime}(s, d)$, то мы могли бы воспользоваться теоремой 1 .А из книги [9], оценка которой отличается от (9) дополнительным множителем $\left(1-e^{-\lambda}\right) / \lambda$, где $\lambda$ - математическое ожидание исследуемой суммы. Однако получаемые таким способом более точные, но «одномерные» результаты типа неравенства (5) бесполезны при изучении совместного распределения величин $N(c, c), \ldots, N(s, c)$.

Д ок а з а т е л ь с т в о н е р а в е н с т в а (2). Рассмотрим произвольное отображение $F:\{0,1\}^{\Gamma} \rightarrow M$, где $M$ - некоторое множество. Очевидно, что

$$
\rho(\mathscr{L}(F(W)), \mathscr{L}(F(\pi))) \leqslant \rho(\mathscr{L}(W), \mathscr{L}(\pi)) .
$$

Возьмем в качестве $F$ отображение, сопоставляющее вектору $\left(x_{a}, a \in \Gamma\right) \in\{0,1\}^{\Gamma}$ вектор $\left(\sum_{a:|\delta|=k} x_{a}, k=c, \ldots, s\right) \in \mathbf{R}^{s-c+1}$. Тогда

a

$$
F(W)=\left(N^{\prime}(k, c), k=c, \ldots, s\right)
$$

$$
\mathscr{L}(F(\pi))=\operatorname{Po}\left(\lambda_{c}(m, n), \ldots, \lambda_{s}(m, n)\right) .
$$

Из теоремы 4 и соотношений (15)-(17) следует (2).

Теорема 1 вытекает непосредственно из неравенства (2), поскольку из ее предположений следует, что $S \rightarrow 0$, a $\lambda_{k}(m, n) \rightarrow \lambda_{k}, k=c, \ldots, s$. 
3. Доказательства для $(s, c)$-совпадений. В этом пункте доказываются оценка (3) теоремы 3 и теорема 2. Начнем с оценки (3). Положим

$$
\tilde{N}(l, c)=\sum_{k=c}^{l}(l-k+1) N^{\prime}(k, c) .
$$

Ясно, что набор $(\tilde{N}(c, c), \ldots, \tilde{N}(s, c))$ является результатом применения к набору $W$ некоторого отображения $F$ из $\{0,1\}^{\Gamma}$ в $\mathbf{R}^{s-c+1}$. Поэтому, согласно (15) и (8),

$\rho\left(\mathscr{L}(\tilde{N}(c, c), \ldots, \tilde{N}(s, c)), \operatorname{CP}\left(\lambda_{c}(m, n), \ldots, \lambda_{s}(m, n)\right)\right) \leqslant \rho(\mathscr{L}(W), \mathscr{L}(\pi)) \leqslant S$.

Определим события

$$
A=\{N(l, c)=\tilde{N}(l, c), l=c, \ldots, s\}, \quad B=\left\{\sum_{k=c+1}^{s+1} N(k, c+1)>0\right\} .
$$

Нетрудно убедиться в том, что $\bar{A} \subseteq B$. Поэтому

$\mathbf{P}(\bar{A}) \leqslant \mathbf{P}(B) \leqslant \sum_{k=c+1}^{s+1} \mathbf{E} N(k, c+1)=\sum_{k=c+1}^{s+1} C_{k-2}^{c-1}(1-R)^{k-c-1} m n R^{c+1}<C_{s}^{c} m n R^{c+1}=T$.

Здесь мы воспользовались неравенством $\mathbf{P}\{Z>0\} \leqslant \mathbf{E} Z$ для случайной величины, принимающей неотрицательные целочисленные значения, выражениями для $\mathbf{E} N(k, c+1)$ и просуммировали биномиальные коэффициенты так же, как и в (6).

Используя неравенство $\rho(\mathscr{L}(U), \mathscr{L}(V)) \leqslant \mathbf{P}\{U \neq V\}$, выполненное для произвольных случайных величин $U$ и $V$, заданных на одном вероятностном пространстве, получаем, что

$$
\rho(\mathscr{L}(s, c), \mathscr{L}(\tilde{N}(c, c), \ldots, \tilde{N}(s, c))) \leqslant T .
$$

По неравенству треугольника из (18) и (19) следует неравенство (3).

Теорема 2 вытекает непосредственно из неравенства (3), поскольку из ее предположений следует, что $S, T \rightarrow 0$, а $\lambda_{k}(m, n) \rightarrow \lambda_{k}$ при всех $k=c, \ldots, s$.

Следствие 1 выводится из теоремы 3 стандартными рассуждениями, использующими тот факт, что функция пуассоновского распределения с ростом к бесконечности его параметра при соответствующей центровке и нормировке сходится к стандартной нормальной функции распределения. Поэтому доказательство следствия 1 мы опускаем.

Следствие 2 вытекает из теоремы 1 и тождества $\{\nu(c)>s\}=\left\{N^{\prime}(c, c)=\cdots=\right.$ $\left.N^{\prime}(s, c)=0\right\}$.

\section{4. Заключительные замечания.}

3 а м е ч а н и е 13. Изложенный метод доказательства может быть использован при анализе предельного поведения числа неполных совпадений частного вида, например, числа $N^{\prime \prime}(s, c)$ таких $(s, c)$-совпадений, в которых нет соседствуюших несовпадений букв.

Теорема 5. Пусть $m, n \rightarrow \infty$, величины $s$ и с постоянны, причем $(s-1) / 2 \leqslant$ $c \leqslant s$, а величины $p_{k}$ и $q_{k}$ меняются так, что выполнены условия (1). Тогда распределение вектора $\left(N^{\prime \prime}(c, c), \ldots, N^{\prime \prime}(s, c)\right)$ сходится $\kappa$ распределению вектора $\left(\pi_{k}^{\prime \prime}+2 \pi_{k-1}^{\prime \prime}+\pi_{k-2}^{\prime \prime}, k=c, \ldots, s\right)$, где $\pi_{c-2}^{\prime \prime}=\pi_{c-1}^{\prime \prime}=0, a \pi_{k}^{\prime \prime}, k=c, \ldots, s,-$ - нез $a-$ висимые случайные величины, распределенные по закону Пуассона с параметрами $\lambda_{k}^{\prime \prime}=\lambda C_{c-1}^{k-c}$.

3 а м е ч а н и е 14. Следует отметить явные аналогии рассмотренной задачи с задачами о числе неполных серий [10] и моменте первого появления не менее $k$ событий пуассоновского потока в интервале заданной длины [11].

Автор признателен А. М. Зубкову за полезные обсуждения полученных результатов и замечания, способствовавшие улучшению изложения. 


\title{
СПИСОК ЛИТЕРАТУРЫ
}

1. Зубков А. М., Михайлов В. Г. Предельные распределения случайных величин, связанных с длинными повторениями в последовательности независимых испытаний. - Теория вероятн. и ее примен., 1974, т. 19, в. 1, с. 173-181.

2. Зубков А.М., Михайлов В.Г. О повторениях $s$-цепочек в последовательности независимых величин. - Теория вероятн. и ее примен., 1979, т. 24, в. 2, с. 267-279.

3. Новак С. Ю. Пуассонова аппроксимация числа длинных «повторов» в случайных последовательностях. - Теория вероятн. и ее примен., 1994, т. 39, в. 4, с. 731-742.

4. Новак С. Ю. Письмо в редакцию. - Теория вероятн. и ее примен., 1995, т. 40, B. 3 , c. 700 .

5. Novak S. Yu. Long match patterns in random sequences. - Siberian Adv. Math., 1995, v. 5, № 3, p. 128-140.

6. Arratia R., Gordon L., Waterman M.S. An extreme value theory for sequence matching. - Ann. Statist., 1986, v. 14, № 3, p. 971-993.

7. Arratia R., Waterman M.S. The Erdös-Rényi strong law for pattern matching with a given proportion of mismatches. - Ann. Probab., 1989, v. 17, № 3, p. 1152-1169.

8. Феллер В. Введение в теорию вероятностей и ее приложения. Т. 1. М.: Мир, 1984, $528 \mathrm{c}$.

9. Barbour A.D., Holst L., Janson S. Poisson Approximation. Oxford: Clarendon Press, 1992, $277 \mathrm{p}$.

10. Arratia R., Gordon L., Waterman M. S. The Erdös-Rényi law in distribution, for coin tossing and sequence matching. - Ann. Statist., 1990, v. 18, № 2, p. 539-570.

11. Зубков A.M. Оценки для сумм конечно-зависимых индикаторов и для момента первого наступления редкого события. - Труды Матем. ин-та им. В. А. Стеклова AH CCCP, 1986, т. 177, с. 33-46.

Поступила в редакцию

5.VII.1999

(C) 2002 г.

РОГОЗИН Б. А. ${ }^{*}$

\section{ТАУБЕРОВЫ ТЕОРЕМЫ ДЛЯ МАЖОРИРУЕМО МЕНЯЮЩИХСЯ УБЫВАЮЩИХ ФУНКЦИЙ ${ }^{1)}$}

\begin{abstract}
Доказываются тауберовы теоремы для мажорируемо меняюшихся убывающихся функций, а также некоторые следствия из них.

Ключевые слова и фразы: тауберова теорема, мажорируемо меняющаяся функция, функция восстановления, возвратное случайное блуждание, время первого возвращения в 0 .
\end{abstract}

Пусть $F$ - распределение на $[0, \infty), \bar{F}(x)=F([x, \infty))$ при $x \geqslant 0$. Функция $\bar{F}(x)$ называется надстепенной на бесконечности убывающей функцией (н. на б. уб. ф.), если при некотором $\lambda(0<\lambda<1) \liminf _{x \rightarrow \infty} \bar{F}(x) / \bar{F}(x \lambda)>0$. Если $\bar{F}-$ н. на $б$. уб. ф., то функция $G(t)=\bar{F}(1 / t), t \geqslant 0$, называется надстепенной в 0 возрастающей функцией (н. в 0 в. ф.), в этом случае $\liminf _{t \rightarrow 0} G(\lambda t) / G(t)>0$.

* Омский филиал Института математики им. С. Л. Соболева СО РАН, ул. Певцова, 13, 644099 Омск, Россия; e-mail: rogozin@iitam.omsk.net.ru

1) Работа выполнена при поддержке Российского фонда фундаментальных исследований (проекты 99-01-01130, 00-15-96178) и INTAS (проект 00-265). 